\title{
Biodegradable Materials with Disulfide-Bridged- Framework Confine Photosensitizers for Enhanced Photo-Immunotherapy
}

\author{
Dongbei Li' \\ Fangman Chen ${ }^{2}$ \\ Cheng Cheng' \\ Haijun $\mathrm{Li}^{3, *}$ \\ Xudong Wei ${ }^{1, *}$
}

'Department of Hematology, Affiliated Tumor Hospital of Zhengzhou University, Henan Cancer Hospital, Zhengzhou City, Henan Province, People's Republic of China; ${ }^{2}$ School of Biomedical Engineering (Suzhou), Division of Life Sciences and Medicine, University of Science and Technology of China, Hefei, 230026, People's Republic of China; ${ }^{3}$ Department of Clinical Laboratory, The First Affiliated Hospital of Zhengzhou University, Zhengzhou, Henan, 450052, People's Republic of China

*These authors contributed equally to this work
Correspondence: Xudong Wei; Haijun Li Email xudongwei@zzu.edu.cn; navylizz@sina.com
Purpose: Photodynamic therapy (PDT) with spatiotemporal controlled and noninvasive advantages has obtained growing attention in cancer treatment. Nevertheless, PDT still suffers from self-aggregation-induced photosensitizer quenching and reactive oxygen species (ROS) scavenging in cancer cells with abundant glutathione (GSH) pools, leading to insufficient performance.

Methods: In this study, we develop a versatile nanocarrier (SSNs) with a disulfide-bondbridged silica framework for enhanced photo-immunotherapy. Such SSNs spatially confine photosensitizers $\mathrm{Ce} 6$ in the matrix to prevent self-aggregation. Under the high GSH level of cancer cells, the disulfide-bond-bridged framework was degradable and triggered the exposure of photosensitizers to oxygen, accelerating the ROS generation during PDT. In addition, GSH depletion via the break of the disulfide-bond increased the ROS level, together resulting in efficient tumor killing outcomes with a considerable immunogenic cell death effect in vitro. Importantly, the SSNs@Ce6 accumulated in the tumor site and exhibited enhanced PDT efficacy with low systemic toxicity in vivo.

Results: The GEN-loaded nanoplatform (Ag-MONs@GEN) showed glutathione-responsive matrix degradation, resulting in the simultaneous controlled release of GEN and silver ions. Ag-MONs@GEN exhibited excellent anti-bacterial activities than Ag-MONs and GEN alone, especially enhancing synergetic effects against four antibiotic-resistant bacteria including Escherichia coli, Pseudomonas aeruginosa, Staphylococcus aureus, and Enterococcus faecalis. Moreover, Ag-MONs@GEN showed good biocompatibility on L929 and HUVECS.

Conclusion: Notably, SSNs@Ce6-mediated PDT completely eradicated 4T1 tumors when combined with the PD-1 checkpoint blockade. Overall, the confinement of photosensitizers in a biodegradable disulfide-bridged-framework provides a promising strategy to unleash the potential of photosensitizers in PDT, especially in combined cancer photo-immunotherapy.

Keywords: photodynamic therapy, glutathione depletion, photosensitizer confinement, degradation, cancer immunotherapy

\section{Introduction}

Cancer has become a primary cause of mortality the global population. The current treatments, including surgery, ${ }^{1}$ chemotherapy, ${ }^{2}$ and radiotherapy, ${ }^{3}$ are limited by off-target toxicity and ineffective outcomes. Photodynamic therapy (PDT) is clinically approved for alternative cancer treatment. ${ }^{4-6}$ Nontoxic photosensitizers are activated by specific light stimuli to produce toxic radical oxygen species (ROS). ${ }^{7-9}$ PDT can selectively ablate cancer cells with spatiotemporal and noninvasive 


\section{Graphical Abstract}

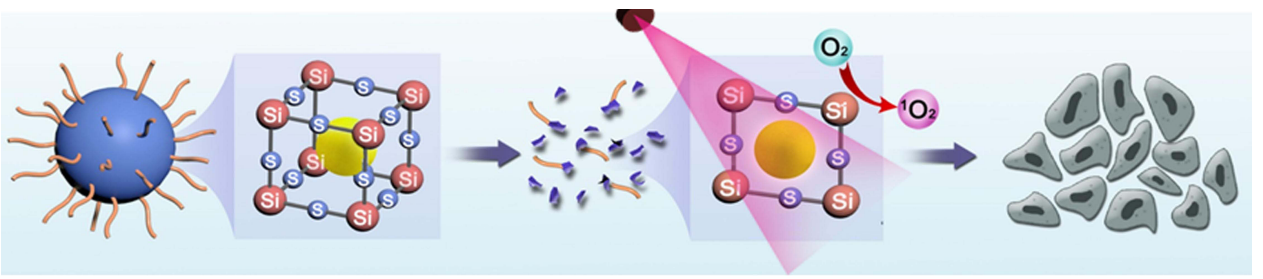

advantages including local irradiation, weakened side effects, and better tolerance by comparing with conventional cancer treatments. ${ }^{10,11}$ In addition, PDT triggered immunogenic cell death (ICD) causes the release of tumorassociated antigens, danger-related molecular patterns (DAMPs), and proinflammatory cytokines, facilitating robust anti-tumor immune responses for combined cancer photo-immunotherapy. ${ }^{12,13,14}$ However, most photosensitizers, including porphyrins, ${ }^{13}$ chlorins, ${ }^{15}$ bacteriochlorins ${ }^{16}$ and phthalocyanines ${ }^{17}$ used for PDT also suffer from poor water solubility, photobleaching, and a lack of target delivery.

Photosensitizers have a tendency to undergo selfaggregation quenching in their excited state, and this compromise the therapeutic effect. ${ }^{18}$ To solve this problem, photosensitizers have been functionalized using an ionic or hydrophilic group to increase the aqueous solubility and prevent $\pi-\pi$ stacking. ${ }^{19}$ In recent years, the 1,3-dipolar cycloaddition of the benzyl azomethine ylide that presented a low tendency to self-aggregate has been adopted by de Souza et al to develop a method. ${ }^{20}$ However, the chemical modification process of photosensitizers was tedious and had a low yield, and this would inhibit their cellular uptake. Increasing evidence has demonstrated that nanocarriers are ubiquitously employed to load photosensitizers through encapsulation or conjugation and deliver them to the target site safely. ${ }^{21}$ The metal-organic frameworks (MOFs) have been used as a promising carrier to isolate or confine photosensitizers to reduce aggregation and photobleaching. ${ }^{22}$ But the host-guest interaction between an MOF and a photosensitizer is limited by their sophisticated physicochemical properties. $^{23}$ Therefore, it is urgent to construct versatile nanocarriers with simple and effective photosensitizer confinement for improved PDT.

A sufficient interaction between oxygen and a photosensitizer is necessary to produce enough cytotoxic ROS for tumor killing. However, in many nanocarriers, the photosensitizer confined in the inner framework is not adequate to access oxygen, and the lifetime of the ROS is very short with a limited distance of action $(<10 \mathrm{~nm}){ }^{7}$ Thus, the development of degradable nanocarriers can increase the exposure of the photosensitizer to oxygen for improved PDT. Moreover, the active glutathione (GSH) associated cellular antioxidant defense system in cancer cells significantly compromises the efficacy of PDT. $^{24}$ The intracellular GSH as a key antioxidant in cancer cells is 100-1000-fold higher than those in normal cells, and GSH exerts a great effect on scavenging ROS, thus weakening the performance of PDT. ${ }^{25}$ Various types of nanocarriers with GSH depletion properties have been reported to amplify oxidative stress and enhance the effectiveness of PDT. ${ }^{26}$ According to these results, it is hypothesized that the development of nanocarriers that integrate matrix degradation with GSH depletion might hold great promise for PDT treatment.

Organosilica nanoparticles (OSNs) have been recently developed as promising drug delivery nanocarriers due to a series of advantages including their tunable morphology and biodegradability, surface-functionalization, and biocompatibility. ${ }^{27-29}$ The principal methods for synthesizing OSNs suffer from a long and complicated extraction to remove surfactants, which lower the final yield. The StÖber method is a promising method to fabricate drugencapsulated silica nanoparticles due to the large scale process without the use of any toxic surfactants. ${ }^{30,31}$ In this case, most of the photosensitizers were dissolvable and stable for encapsulation into the matrix of silica to prevent self-aggregation. Previous researches have reported that OSNs exhibit GSH-responsive biodegradability with controlled matrix degradation for controlled drug release. ${ }^{32}$ We believe that such a GSH-responsive nanocarrier might prevent self-aggregation during the high encapsulation of photosensitizers and show matrix 
degradation and GSH depletion for efficient and secure PDT.

This research proposes a facile strategy to fabricate disulfide-bond-bridged organosilica nanoparticles (SSNs) to delivery photosensitizers for highly efficient cancer photo-immunotherapy. Regardless of the physicochemical properties of the photosensitizers, they have demonstrated efficient encapsulation into the framework of versatile SSNs. The chlorin e6 (Ce6) is chosen as a model photosensitizer to evaluate drug loading and matrix-degradation under the GSH-enriched tumor microenvironment. ${ }^{33-35}$ The well-confined Ce6 in Ce6@SSNs efficiently absorbed light and avoided aggregation-induced quenching. Such a degradable matrix increased the exposure of the photosensitizers to oxygen. In addition, the Ce6@SSNs underwent a structure disruption caused by the GSH-based disulfide bond cleavage, leading to GSH depletion and ROS enhancement for amplified cancer cell death. In vitro and in vivo studies validated the efficiency and safety of PDT. A further combination with anti-PD-1 effectively eradicated the tumor on a murine breast cancer model.

\section{Experimental}

\section{Materials}

Sigma-Aldrich (St. Louis, MO, United States) provided tetraethyl orthosilicate (TEOS), Chlorin e6 (Ce6), aluminum-tri-sec-butoxide, and and $\gamma$-chloropropyl trimethoxysilane (CP). Beijing Chemical Reagent Co. (Beijing China) supplied the Bis[3-(triethoxysilyl)propyl]tetrasulfide (BTESPT), hydrogen peroxide (30\% $\mathrm{H} 2 \mathrm{O} 2)$, and anhydrous ethanol. GIBCO (Carlsbad, CA, United States) offered the [3-(4, 5-dimethylthiazol-2-yl)-2, 5-diphenyl] tetrazolium bromide (MTT), Dulbecco's Modified Eagle Medium (DMEM), fetal bovine serum (FBS), penicillin-streptomycin, and trypsin $(10,000 \mathrm{U} / \mathrm{mL})$. Matrigel was purchased from Corning Inc. (Billerica, MA, United States). The Thermo Scientific Pierce Ellman (DTNB), reduced GSH assay kit, 4, 6-diamidino-2-phenylindole (DAPI), 1,3Diphenylisobenzofuran (DPBF), and 2',7'-dichlorofluorescin diacetate (DCFH-DA) were obtained from Thermo Fisher Scientific (Waltham, MA, United States). Assay kits for determining alkaline phosphatase (ALP), alanine aminotransferase (ALT), aspartate aminotransferase (AST), blood urea nitrogen (BUN) and creatinine (CRE) were purchased from the Nanjing Jiancheng Bioengineering Institute (Nanjing, Jiangsu, China). 4T1 mouse breast cancer cells purchased from the American Type Culture
Collection (ATCC) were used in the experiments. The above reagents were directly applied with no purification.

\section{Preparation of SSNs}

The SSNs was prepared using the typical StÖber method below. The mixture of an ammonia aqueous solution $(0.75$ $\mathrm{mL})$ with water-alcohol (2: $25.0 \mathrm{~mL})$ was performed. Then a certain amount $(0.5 \mathrm{~mL})$ of TEOS, BTESPT $(0.2 \mathrm{~mL})$, and aluminum-tri-sec-butoxide $(0.01 \mathrm{~mL})$ were added dropwise into the above mixture. The reaction system was stirred at room temperature for the overgrowth. In the end, ethyl acetate was put to precipitate the generated SSNs. The resultant SSNs were purified and collected by washing with ethanol and water. The SNs was prepared in a similar manner to the above method except in the absence of BTESePD. The photosensitizer was mixed with saline to prepare the SSNs@FL, SSNs@AD, and SNs@Ce6.

\section{Characterization of SSNs}

The morphology was observed on a transmission electron microscope (TEM) (JEOL, Ltd., Japan) and a scanning electron microscope (SEM) (FESEM, S4800, Hitachi Co. Ltd., Tokyo, Japan). The fluorescence spectroscopy was recorded applying the Shimadzu RF-5301 PC spectrophotometer. The size distribution and Zeta potential was determined with a Nano-ZS 90 Nanosizer (Malvern Instruments Ltd., Worcestershire, United Kingdom).

\section{Extracellular GSH Depletion Assay}

The mixture of GSH solution with the SSNs@Ce6 (0.5 $\mu \mathrm{g}$ / $\mathrm{mL}$ Ce6) was performed. The mixtures were gently shaken at $37^{\circ} \mathrm{C}$, and then the resulting solution was filtered with a $0.22 \mu \mathrm{m}$ syringe filter for reacting with the DTNB. The UV-vis absorbance at $421 \mathrm{~nm}$ was recorded.

\section{Extracellular ROS Detection}

1.3-Diphenylisobenzofuran (DPBF) was adopted as a singlet oxygen fluorescent label to evaluate the PDT efficacy of the preparations. Briefly, the SSNs@Ce6, SNs@Ce6, and Ce6 with/without $10 \mathrm{mM}$ of GSH at the same concentration $(0.5 \mu \mathrm{g} / \mathrm{mL} \mathrm{Ce} 6)$ were rapidly mixed with DPBF. After 660-nm laser irradiation $\left(100 \mathrm{~mW} \mathrm{~cm}^{-2}\right)$ was applied for 10 minutes, the UV-vis absorption spectra of the solutions were recorded. 


\section{Intracellular GSH Depletion Assay}

The 24-hour seeding of 4T1 cells into a six-well plate and cultured was made. After 12-hour incubation with SSNs@Ce6 $(0.5 \mu \mathrm{g} / \mathrm{mL}$ Ce6), the cells were washed with phosphate buffered saline (PBS) and analyzed with the GSH assay kit on basis of the manufacturer's protocol.

\section{Intracellular ROS Generation Assay}

The intracellular ROS generation was researched using a DCFH-DA probe with laser irradiation. The overnight culture of 4T1 cells was made in 24-well plates. After being treated with SSNs@Ce6, SNs@Ce6, and Ce6 (0.5 $\mu \mathrm{g} / \mathrm{mL}$ Ce6), the $4 \mathrm{~T} 1$ cells were incubated for $4 \mathrm{~h}$, then washed twice with PBS (pH 7.4). The 4T1 cells were incubated with DCFH-DA for $2 \mathrm{~h}$, then washed with PBS and exposed to 66- $\mathrm{nm}$ laser irradiation $(100 \mathrm{~mW}$ $\mathrm{cm}^{-2}, 10 \mathrm{~min}$ ). Flow cytometry and fluorescent imaging for quantitative and qualitative detection, respectively were adopted to measure the fluorescence intensity of the DCF immediately.

\section{Cytotoxicity, ICD Effect and DC Maturation in vitro}

The seeding of cells into 96-well culture plates with 5000 cells per well was made, next to the overnight culture for fully attachment. Then, various final concentrations of the formulations were adopted to treat the cells. After $24 \mathrm{~h}$, the cell viabilities were measured using MTT.

The seeding of $4 \mathrm{~T} 1$ cells into a 24 -well plate $\left(5 \times 10^{4}\right.$ cells/well) was made, next to 24-hour incubation. Then the cells were treated with different formulations with/without light irradiation. The cells were collected and stained with anti-CRT antibody for measuring the calreticulin (CRT) expression. The cell culture supernatants were analyzed using an ELISA kit for detection of HMGB1 secretion.

For the detection of dendritic cell maturation, DCs from the mouse bone marrow were co-incubated with the treated $4 \mathrm{~T} 1$ cells for $24 \mathrm{~h}$. Then the DCs were collected and stained with anti-CD11c-FITC, anti-CD80-PE, and anti-CD86-APC for the FACS analysis.

\section{In vivo Experiments}

This study was approved by the Life Science Ethics Review Committee of Zhengzhou University (Zhengzhou,Henan, China). Animal experimentation protocols were in accordance with the guide lines of the Institutional Committee for the Ethics of Animal Care and Treatment at Zhengzhou University. The subcutaneous injection of $4 \mathrm{~T} 1$ cells $\left(5 \times 10^{5}\right)$ into the left flank of the $\mathrm{BALB} / \mathrm{C}$ mic was conducted. When the tumor volume was about $100 \mathrm{~mm}^{3}$, the tumor bearing mice were administered an intravenous injections of the formulations at a $\mathrm{Ce} 6$ dose of $2 \mathrm{mg} / \mathrm{kg}$. All of the drug treatments were monitored every three days, and the tumor volume and body weight were measured in the meantime. After 21 days, all of the mice were sacrificed on day 22 . The tumors were weighed. Hematoxylin and eosin (H\&E) were used to stain the main organs (liver, spleen, kidneys, hearts, and lungs). The biosafety was evaluated using body weight, pathological changes, and serum biochemistry indexes.

\section{Statistical Analysis}

All of the experiments were repeated at least three times, and the outcomes are displayed as means \pm standard deviations. A contrast between the groups was calculated using a Student's $t$-test (two groups) or Bonferroni's post hoc test (three groups or more). The analysis of data was performed on SPSS software. Differences were regarded statistically significant when the p-values were less than 0.05 .

\section{Results and Discussion}

The disulfide-bond-bridged SSNs were then fabricated using a modified Stöber method. Briefly, tetraethyl orthosilicate (TEOS) and BTESPT as co-precursors were catalyzed using ammonium hydroxide in an alcohol-water mixture at room temperature. The sulfide contents in the hybrid SSNs were tunable by the mass ratio of TEOS to BTESPT. In the present study, the mass ratio of TEOS to BTESPT was defined to be $4: 1$. In addition, the inorganic silica nanocarrier ( $\mathrm{SNs}$ ) was also synthesized in parallel for comparison to demonstrate the advantages of the disulfide -bond-bridged SSNs for enhanced PDT. The transmission electron microscopy (TEM) imaging revealed a uniform spherical morphology of the SSNs with average diameters $(\sim 70 \mathrm{~nm})$ (Figure 1A). This result was consistent with the results of the scanning electron microscopy (SEM) (Figure 1B). The dynamic light scattering (DLS) measurements gave a number averaged size of $80.5 \pm 6.1$ $\mathrm{nm}$ (Figure S1). The SNs showed comparable morphologies as the SSNs (Figure S2). The elemental mappings showed the uniform distributions of the $\mathrm{Si}, \mathrm{O}$, and $\mathrm{Se}$ elements within the SSNs matrices (Figure 1C), while the inductively coupled plasma optical emission spectrometry showed sulfide content (8.7\%) and aluminum 

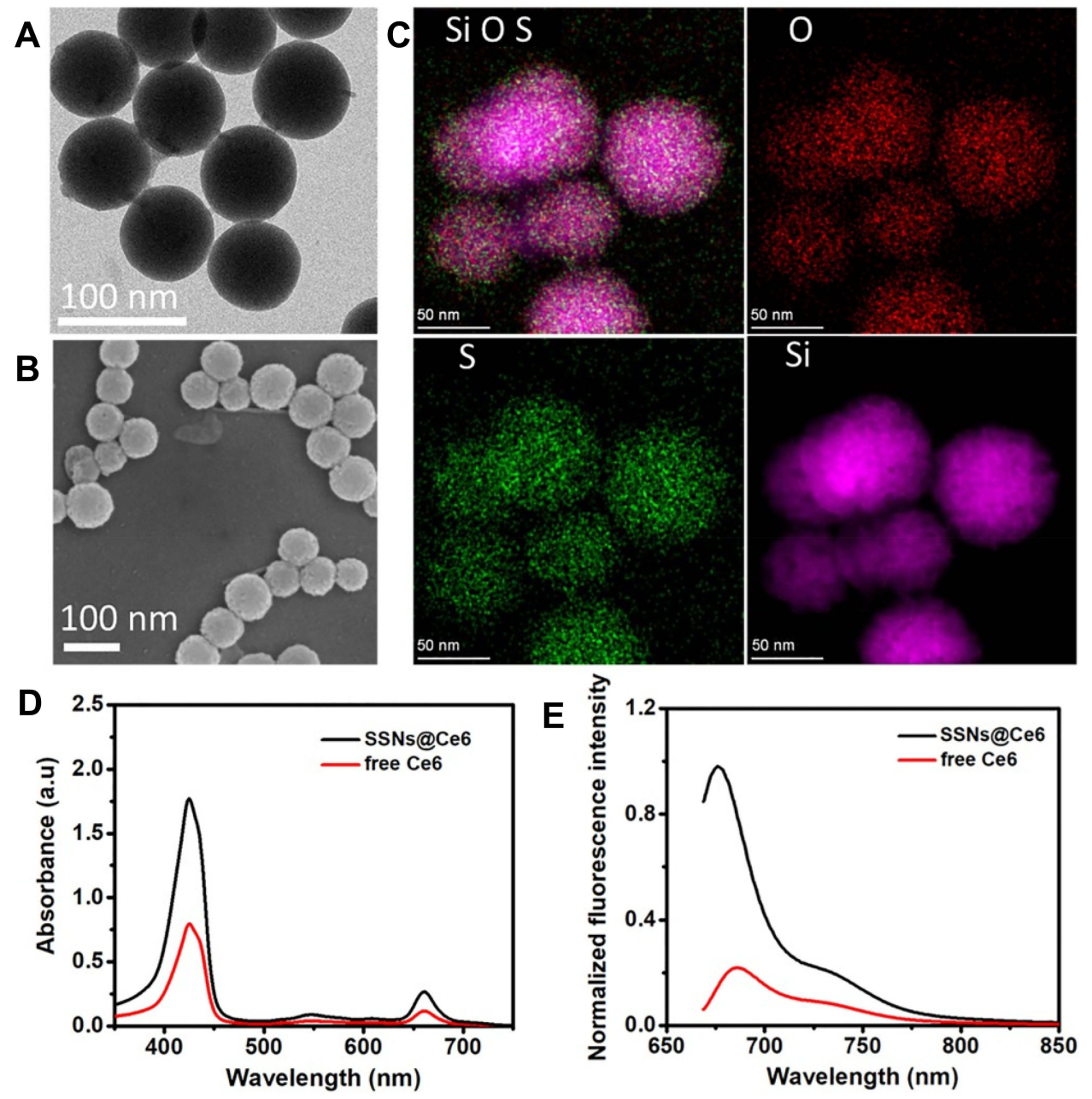

Figure I Preparation and characterization of SSNs.

Notes: (A) TEM and (B) EDX mapping images of SSNs. (C) EDX mapping images of SSNs. (D) absorbance spectra of free Ce6 and SSNs@Ce6. (E) Emission spectra of Ce6 and SSNs@Ce6.

Abbreviations: TEM, transmission electron microscopy; EDX, energy dispersive X-ray spectroscopy; SSNs, disulfide-bond-bridged organosilica nanoparticles; FL, fluorescein; SSNs@FL, fluorescein-loading disulfide-bond-bridged organosilica nanoparticles; AD, adriamycin; SSNs@AD, adriamycin-loading disulfide-bond-bridged organosilica nanoparticles; Ce6, chlorin e6; SSNs@Ce6, chlorin e6-loading disulfide-bond-bridged organosilica nanoparticles.

content $(0.1 \%)$. The zeta potential measurements revealed a negative surface charge of the SSNs $(-12.1 \pm 0.93 \mathrm{mV})$ (Figure S3).

To judge the effect on the UV-visible absorption of photosensitizer Ce6 in SSNs, the UV-visible absorption test was performed on the Ce6 and SSNs@Ce6 (Figure 1D). We found that the SSNs@Ce6 enhanced absorption peak in the range of 400-800 $\mathrm{nm}$. Taking into account the light penetration, red light $660 \mathrm{~nm}$ is used for subsequent photodynamic therapy. Photosensitizers suffer from fluorescence aggregation-caused quenching (ACQ) due to excitonic coupling when they pack together at high concentrations or in a solid state. We suspected that the SSNs as a versatile carrier encapsulated and confined the photosensitizers in the matrix to circumvent ACQ. Fluorescein (FL), Adriamycin (AD), and Chlorin e6 (Ce6) are typical aggregation-induced fluorescence quenching fluorophores with neutral, positive, and negative charges, respectively. The FL, AD, and Ce6 were effectively encapsulated into the framework obtained SSNs@FL, SSNs@AD, and SSNs@Ce6, respectively. The strong fluorescence emissions of the SSNs@FL, SSNs@AD, and SSNs@Ce6 (Figures 1E and S1) confirmed that fluorophore encapsulated and confined in the matrix to prevent fluorescence aggregation-caused quenching.

Given Ce6 is widely used in PDT, SSNs@Ce6 was used as a model formulation to validate the enhanced 
photodynamic therapy. The degradation behavior of SSNs@Ce6 was investigated in the media mimicking tumor micro-environment (10 mM GSH). The TEM images showed that after one day of incubation, the SSNs@Ce6 underwent rapid degradation into fragments similar to two-dimensional sheets (Figure 2A). In contrast, the SNs@Ce6 only showed a stable structure in the mimicking media (Figure 2B). Our previous studies had confirmed that that the disulfide-bond bridged matrix could be reductively broken by GSH. In addition, reductive GSH was consumed. Then, the amount of GSH in the mimicking media was measured after incubation with SSNs@Ce6 for 24 h. As shown in Figure 2C, the content of GSH was decreased in a concentration dependent manner, indicating excellent GSH depletion. In contrast, SNs@Ce6 did not consume the GSH, consistent with the results of degradation.

GSH, a significant intracellular antioxidant, exerts a great effect on scavenging the ROS produced by PDT. We further investigated that degradation and GSH depletion influence on the PDT efficiency. ROS generated from PDT were detected using 1,3-Diphenyl-2-benzofuran (DPBF) (Figure 2D), and the UV-vis absorbance of the DPBF incubated with SNs@Ce6 was decreased significantly. However, the UV-vis absorbance of the DPBF displayed stain stability in the presence of $10 \mathrm{mM} \mathrm{GSH}$ due to the ROS scavenging by GSH. Conversely, after red light irradiation, the UV-vis absorbance of the DPBF incubated with SSNs@Ce6 rapidly decreased compared with that of SNs@Ce6 in the presence of 10 mM GSH. The results indicated that SSNs@Ce6 consumed GSH to prevent ROS scavenging by GSH and facilitated PDT efficacy. In addition to the GSH depletion to inhibit ROS scavenging, the degradation may mediate exposure of inner $\mathrm{Ce} 6$ to oxygen to increase ROS generation. SNs@Ce6 and SSNs@Ce6 showed higher UV-vis absorbances of DPBF than the free $\mathrm{Ce} 6$ at the same concentration, and this verified that $\mathrm{Ce} 6$ in the matrix prevented aggregation-induced quenching to increase ROS generation. In summary, the SSNs@Ce6 enhanced their PDT efficiency, and this was attributed to photosensitizer confinement, the degradation mediating exposure of the photosensitizer to increase ROS generation, and GSH depletion to prevent ROS scavenging.

The potent fluorescence of SSNs@Ce6 was suitable for real-time imaging. The cellular uptake of SSNs@Ce6 was evaluated with confocal laser scanning microscopy (CLSM) subsequently. After the 4T1 cells were incubated with SSNs@Ce6 for 24 h, the red fluorescence signal of SSNs@Ce6 gradually grew with incubation time and showed a much higher fluorescence than the free Ce6 (Figure $3 \mathrm{~A}$ and $\mathrm{B}$ ). The quantitative analysis using flow

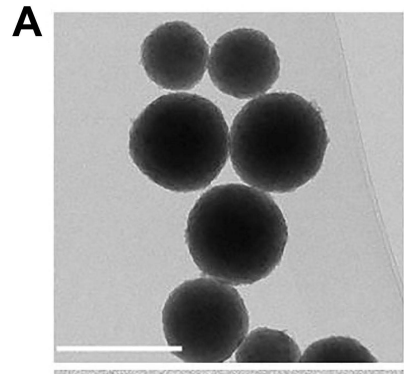

B

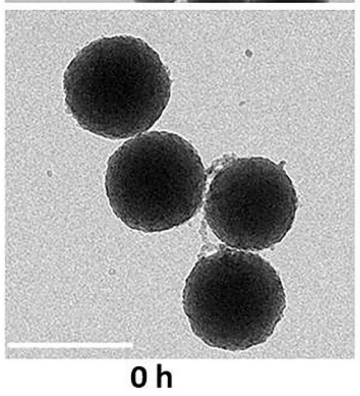

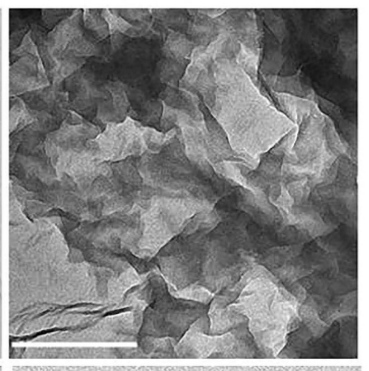

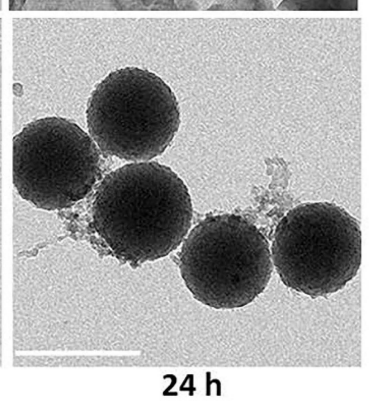

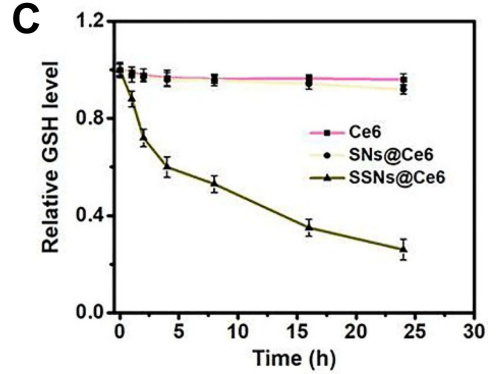

D

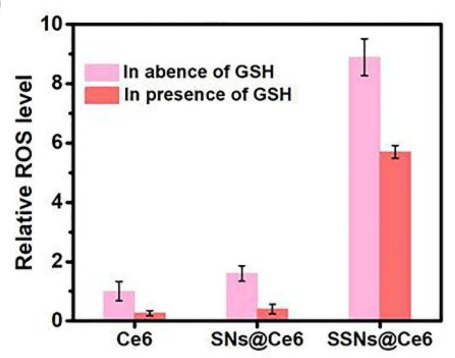

Figure 2 Extracellular GSH depletion assay and extracellular ROS detection.

Notes: Degradation of (A) SSNs@Ce6 and (B) SNs@Ce6 in 10 mM GSH solution. (C) GSH depletion of free Ce6, SNs@Ce6 and SSNs@Ce6. (D) ROS generation of free Ce6, SNs@Ce6 and SSNs@Ce6 upon660 nm light irradiation. Scar bar repesents $100 \mu \mathrm{m}$.

Abbreviations: GSH, glutathione; ROS, radical oxygen species; Ce6, chlorin e6; SNs, inorganic silica nanocarrier; SNs@Ce6, chlorin e6-loading inorganic silica nanocarrier; SSNs@Ce6, chlorin e6-loading disulfide-bond-bridged organosilica nanoparticles. 

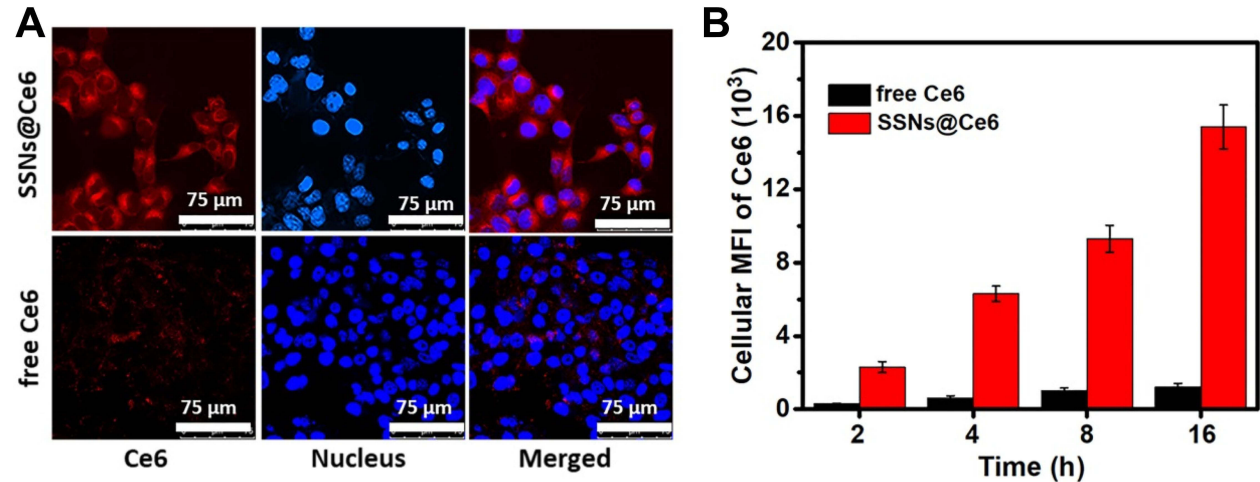

Figure 3 Fluorescence imaging and quantitative analysis in 4TI.

Notes: (A) Fluorescence imaging of cellular uptake of SSNs@Ce6 and free Ce6 in 4TI. (B) Quantitative analysis of the internalization of SSNs@Ce6 and free Ce6 in 4TI. Abbreviations: Ce6, chlorin e6; SSNs@Ce6, chlorin e6-loading disulfide-bond-bridged organosilica nanoparticles.

cytometry verified a 15-fold higher uptake of SSNs@Ce6 than that of free $\mathrm{Ce} 6$ in the $4 \mathrm{~T} 1$ cells (Figure 3B). These results confirmed the efficient cellular uptake of SSNs@Ce6.

The ROS production of SSNs@Ce6 in the 4T1 cells was further analyzed using fluorescence imaging and flow cytometry, and the cellular GSH depletion was evaluated as well. A commercial kit measurement was adopted to measure the depletion by SSNs@Ce6. According to Figure 4A, after incubation with SSNs@Ce6, greater than 66.4\% of the GSH was consumed in the 4T1 cells. However, the free Ce6 and SNs@Ce6 showed a fewer influence on the intracellular GSH level. These results verified that the disulfide-bond containing SSNs@Ce6 played a key role in decreasing the intracellular GSH concentration. A fluorescent indicator 2', 7'-dichlorofluorescein diacetate (DCFH-DA) was adapted to further measure the intracellular ROS production. The quantitative results were obtained using flow cytometry (Figure 4B). Under 660 $\mathrm{nm}$ of light irradiation $\left(100 \mathrm{~mW} / \mathrm{cm}^{2}, 10 \mathrm{~min}\right)$, the fluorescence intensity (MFI) in the group was 6.3-fold and 2.3fold higher than that of the free $\mathrm{Ce} 6$ and $\mathrm{SNs} @ \mathrm{Ce} 6$ groups, respectively, verifying that the SSNs@Ce6 increased ROS generation. A similar phenomenon was
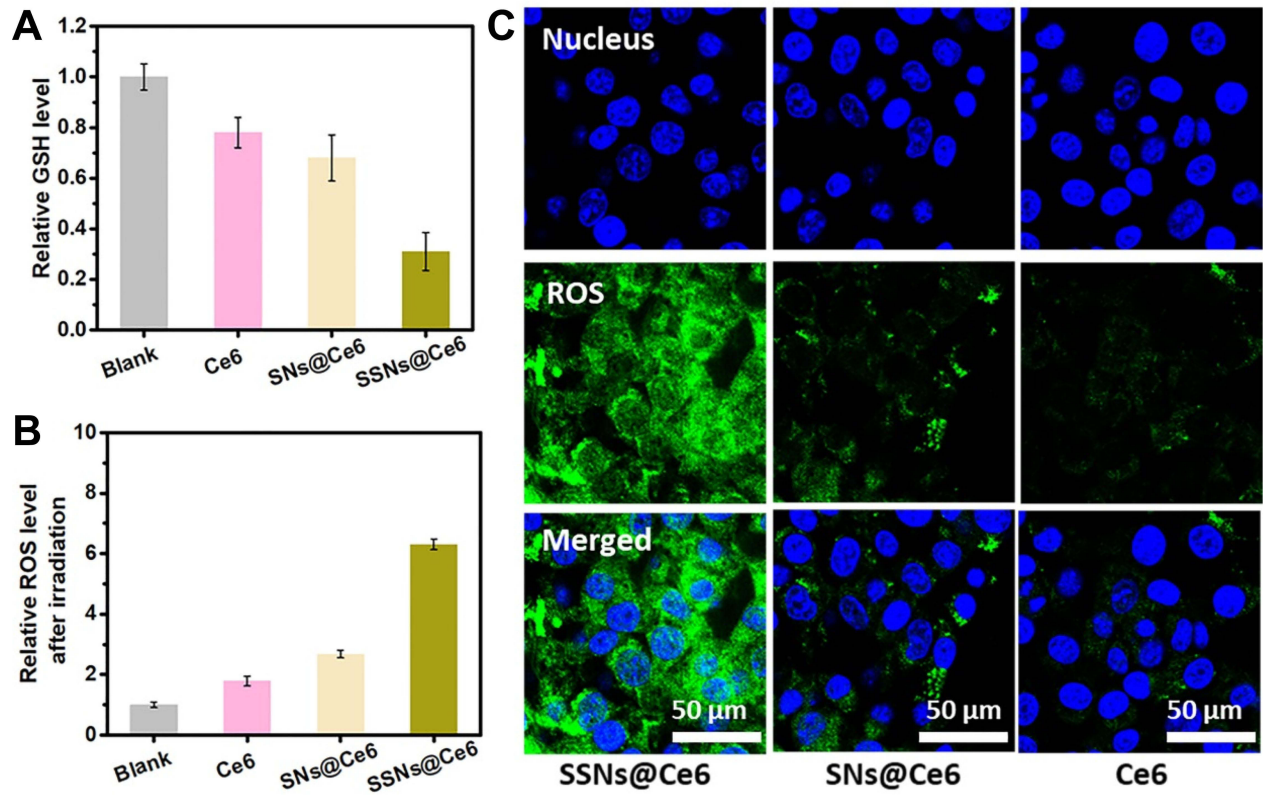

SSNs@Ce6

SNs@Ce6

Ce6

Figure 4 Intracellular GSH depletion ROS generation assay in 4TI.

Notes: Quantitative analysis of (A) GSH depletion and (B) ROS generation in 4TI cells. (C) Fluorescence imaging of ROS generation in 4TI cells. Abbreviations: GSH, glutathione; ROS, radical oxygen species. 
observed in the fluorescence imaging (Figure 4C). The enhanced ROS generation of SSNs@Ce6 could be caused by the decrease in ROS consumed by GSH depletion and the isolation effect and exposure of the photosensitizer to an increase in ROS generation.

For the evaluation of the therapeutic efficacy of SSNs@Ce6, the cytotoxicity was evaluated using an MTT assay. The 4T1 cells were treated with different concentrations of formulations. According to Figure S5, ignorable toxicities were observed in all the groups without laser irradiation, indicating a good biocompatibility. In addition, under laser irradiation $\left(660 \mathrm{~nm}, 50 \mathrm{~mW} / \mathrm{cm}^{2}\right.$ for 10 min), SSNs@Ce6 showed a remarkable cytotoxicity in a dosage dependent way (Figure 5A), and this was greatly higher cytotoxicity than that of the free $\mathrm{Ce} 6$ and SNs@Ce6. These results revealed that SSNs@Ce6 promoted highly efficient PDT in vitro.

Previous research has reported that PDT could evoke immunogenic cell death (ICD) effects with the characters such as the high expression of calreticulin (CRT) on the surface of dying cancer cells and the release of chromatinbinding protein high mobility group B1 (HMGB1). We characterized that the PDT of the SSNs@Ce6 mediated ICD and the immune activation (Figure 5B-D). Under light irradiation, the SSNs@Ce6 induced a higher percentage of CRT-positive cells (Figure 5B), a higher level of HMGB1 (Figure 5C), and a greater DC maturation (Figure 5D) in vitro than that of free Ce6 and SNs@Ce6. This result indicated that SSNs@Ce6 based on the combination of enhanced ROS generation and GSH depletion amplified the PDT and ICD efficiency. These results confirmed that PDT of SSNs@Ce6 promoted ICD to expose tumor antigens and danger signals for immune activation.

One challenge of PDT is achieving a long blood circulation time and a sufficient cumulation of photosensitizers in a tumor. The pharmacokinetic profiles of SSNs@Ce6, SNs@Ce6, and Ce6 in blood were monitored by measuring the fluorescence signal of $\mathrm{Ce} 6$ in serum (Figure 6A). The SSNs@Ce6 exhibited a remarkably longer elimination half-life $\left(\mathrm{T}_{1 / 2}, 18.4 \mathrm{~h}\right)$ that was higher than that of free $\mathrm{Ce} 6$ $\left(\mathrm{T}_{1 / 2}, 4.1 \mathrm{~h}\right)$. This extended blood retention suggested that the PEGylated nanocarrier conferred colloid stability and reduced the uptake of the host immune system in vivo. The biodistribution of SSNs@Ce6 treated 4T1 tumor-
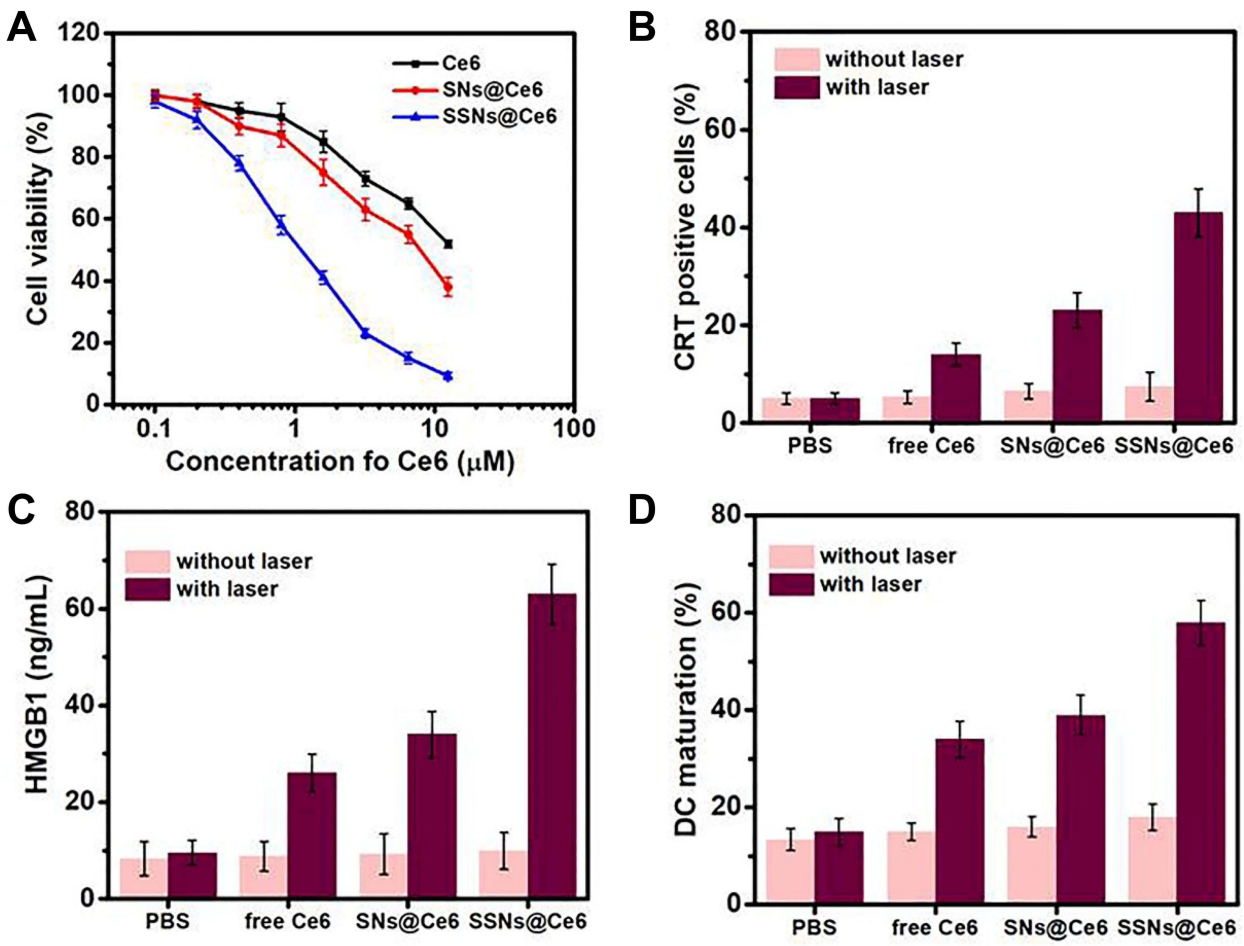

Figure 5 Cytotoxicity, ICD effect and DC maturation in vitro.

Notes: (A) Cytotoxicity of free Ce6, SNs@Ce6 and SSNs@Ce6 against 4TI cells with light irradiation $\left(0.1\right.$ W cm ${ }^{-2}, 10$ min) after 24 h incubation. (B) Percentage of CRTpositive cells and (C) amount of released HMGBI after $24 \mathrm{~h}$. (D) Percentage of mature DCs $\left(\mathrm{CDI} / \mathrm{c}^{+} \mathrm{CD} 80^{+} \mathrm{CD} 86^{+}\right)$after co-incubation with $4 \mathrm{TI}$ cells with different treatments for $24 \mathrm{~h}$.

Abbreviations: Ce6, chlorin e6; SNs, inorganic silica nanocarrier; SNs@Ce6, chlorin e6-loading inorganic silica nanocarrier; SSNs@Ce6, chlorin e6-loading disulfide-bondbridged organosilica nanoparticles; CRT, calreticulin. 

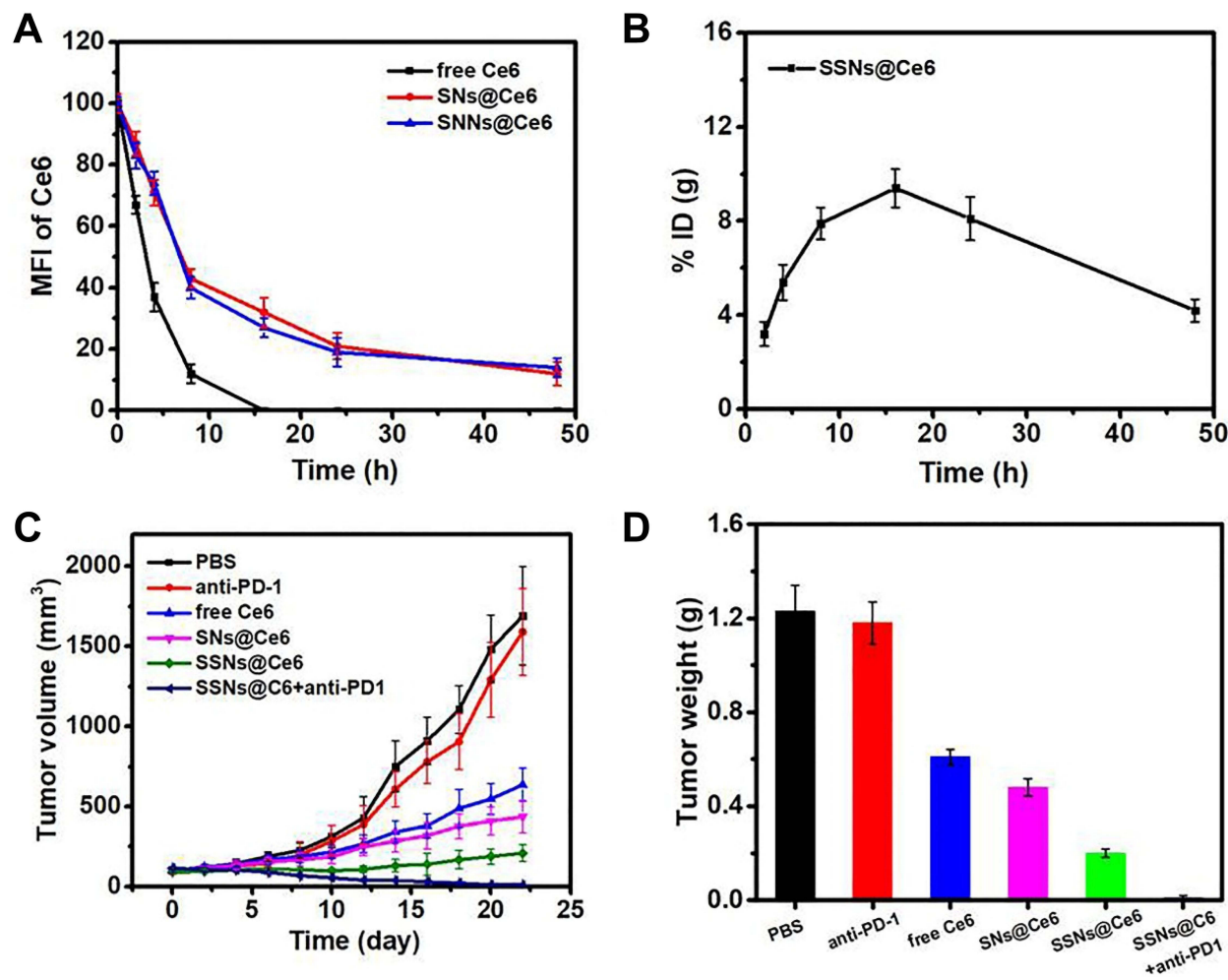

Figure 6 In vivo experiments.

Notes: (A) Blood circulation time of free Ce6, SNs@Ce6 and SSNs@Ce6 in 4TI-tumor-bearing mice. (B) Accumulation of SSNs@Ce6 in tumor tissue. (C) Tumor volume and (D) tumor weight after treating with formulations in 4TI-tumor-bearing mice.

Abbreviations: Ce6, chlorin e6; SNs, inorganic silica nanocarrier; SNs@Ce6, chlorin e6-loading inorganic silica nanocarrier; SSNs@Ce6, chlorin e6-loading disulfide-bondbridged organosilica nanoparticles.

bearing mice was evaluated by determining the silicon ( $\mathrm{Si})$ content of the tumor tissue and major organs at $16 \mathrm{~h}$ post intravenous injection (Figure 6B). The high accumulation of $\mathrm{Si}$ at the tumor site indicated that SSNs@Ce6 was efficiently delivered to the tumor tissue. Enrichment in the reticuloendothelial system, including the liver and spleen, was observed (Figure S6), indicating that the reticuloendothelial system may involve the metabolism of SSNs@Ce6 for clearance.

Next, the therapeutic effect of SSNs@Ce6 in vivo was studied. The 4T1 tumor-bearing mice were treated with PBS, anti-PD-1+L, SSNs@Ce6, Ce6+L, SNs@Ce6+L, SSNs@Ce6+L, or SSNs@Ce6+anti-PD-1+L (Ce6=2 mg/ $\mathrm{kg}$, anti-PD-1=3 mg/kg), and some of the mice received light irradiation $\left(660 \mathrm{~nm}, 0.2 \mathrm{~W} / \mathrm{cm}^{2}, 10 \mathrm{~min}\right) 16 \mathrm{~h}$ post injection. According to Figure $6 \mathrm{C}$ and $\mathrm{D}$, treatment with PBS, anti-PD-1+L, and SSNs@Ce6 led to negligible tumor development inhibition. In contrast, SSNs@Ce6+L showed more efficient tumor growth inhibition than $\mathrm{Ce} 6$ +L and SNs@Ce6+L, demonstrating the advantage of the combination of enhanced ROS generation and GSH depletion for enhanced PDT. In addition, extensive evidence has demonstrated that ICD generating tumor-associated antigens can produce antitumor immunological responses to ablate tumor cell residues. Considering SSNs@Ce6+L inducing ICD effects in the 4T1 cells, the combination of PDT with anti-PD-1 was performed. Compared with treatment by the SSNs@Ce6+L groups, the 4T1 tumor bearing mice treated with SSNs@Ce6+anti-PD-1+L groups completely ablated the tumor, indicating the effective combination of photo-immunotherapy.

The potential side effects of cancer therapy are a great concern for application. The bodyweight alterations after treatment were similar to those of the PBS group as contra (Figure 7A), suggesting no greatly systemic toxicity. The systemic toxicity was evaluated by adopting the blood biochemical indices and H\&E staining of organ slices. The functions of the liver were evaluated by measuring the alanine aminotransferase (ALT), aspartate aminotransferase (AST), and lactate dehydrogenase (LDH) levels in the serum. The functions of the kidney and heart, respectively were evaluated with blood urea nitrogen (BUN) and the creatinine kinase $(\mathrm{CK})$ level in the serum. By comparing with those in the PBS group, the treatment groups 

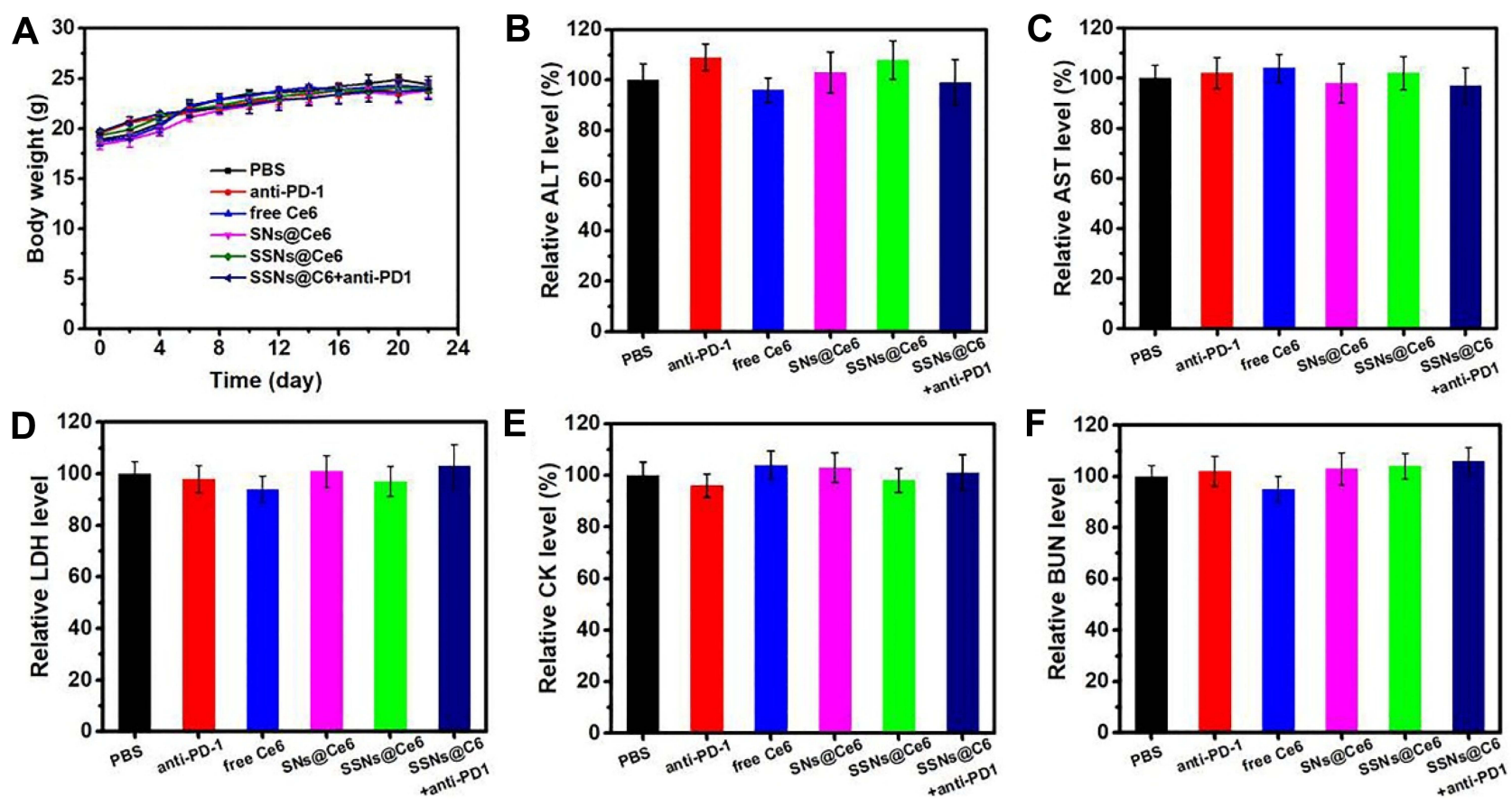

Figure 7 Biosafety profile.

Notes: (A) Body weight, (B) ALT, (C) AST, (D) LDH, (E) CK, and (F) BUN.

Abbreviations: ALT, alanine aminotransferase; AST, aspartate aminotransferase; BUN, blood urea nitrogen; LDH, lactate dehydrogenase; CK, creatinine kinase.

showed no abnormal alterations in the ALT, AST, LDH, BUN, and CK levels (Figure 7B-F). Moreover, all of the treatment groups did not display obvious pathological alterations in the heart, liver, spleen, lung, and kidney (Figure S7). These results collectively corroborated that all of the treatment groups exhibited no great toxicity, suggesting safety and efficient PDT.

\section{Conclusions}

In summary, we constructed a disulfide-bond-bridged SSNs as a versatile nanocarrier to encapsulate photosensitizers for combined cancer photo-immunotherapy. The encapsulated Ce6 in SSNs@Ce6 efficiently prevented self-aggregation-induced quenching and facilitated the exposure of inner photosensitizers to oxygen for enhanced ROS generation. In addition, the matrix of the disulfidebond-bridged matrix was degradable to consume excess abundant intracellular GSH and increase the ROS level, resulting in efficient PDT potency and evoking a robust ICD effect in vitro. After systemic administration, the SSNs@Ce6 exhibited long blood circulation and high tumor accumulation behavior, and this enhanced the PDT efficacy on 4T1-breast tumor bearing mice. With the aid of anti-PD-1, SSNs@Ce6 mediated PDT eradicated the tumor without systemic toxicity. This work suggests a design for a degradable nanocarrier with a disulfide-bond-bridged framework for the confinement of photosensitizers that provides a promising and safe strategy for cancer PDT, particularly for combination photo-immunotherapy.

\section{Acknowledgments}

This study was supported by Henan Medical Science and Technology Project (NO.LHGJ20190647, SBGJ202003 012).

\section{Disclosure}

The authors declare that the research was performed without any commercial or financial relationships that could be construed as hidden conflicts of interest.

\section{References}

1. Olov N, Bagheri-Khoulenjani S, Mirzadeh H. Combinational drug delivery using nanocarriers for breast cancer treatments: a review. $J$ Biomed Mater Res A. 2018;106(8):2272-2283. doi:10.1002/jbm.a.3 6410

2. Zhang RX, Wong HL, Xue HY, et al. Nanomedicine of synergistic drug combinations for cancer therapy - strategies and perspectives. $J$ Control Release. 2016;240:489-503. doi:10.1016/j.jconrel.2016.06. 012

3. Huang RX, Zhou PK. DNA damage response signaling pathways and targets for radiotherapy sensitization in cancer. Signal Transduct Tar. 2020;5(1):60. doi:10.1038/s41392-020-0150-x 
4. Alsaab HO, Alghamdi MS, Alotaibi AS, et al. Progress in clinical trials of photodynamic therapy for solid tumors and the role of nanomedicine. Cancers. 2020;12:10. doi:10.3390/cancers12102793

5. Jia D, Ma X, Lu Y, et al. ROS-responsive cyclodextrin nanoplatform for combined photodynamic therapy and chemotherapy of cancer. Chinese Chem Lett. 2021;32(1):162-167. doi:10.1016/j.cclet.2020. 11.052

6. Chen S, Liu Y, Liang R, et al. Self-assembly of amphiphilic peptides to construct activatable nanophotosensitizers for theranostic photodynamic therapy. Chinese Chem Lett. 2021. doi:10.1016/j. cclet.2021.06.041

7. Li X, Lee S, Yoon J. Supramolecular photosensitizers rejuvenate photodynamic therapy. Chem Soc Rev. 2018;47(4):1174-1188. doi:10.1039/c7cs00594f

8. Zhou L, Zhao J, Chen Y, et al. MoS2-ALG-Fe/GOx hydrogel with Fenton catalytic activity for combined cancer photothermal, starvation, and chemodynamic therapy. Colloids Surf B Biointerfaces. 2020;195:111243. doi:10.1016/j.colsurfb.2020.111243

9. Luo K, Wu H, Chen Y, et al. Preparation of Bi-based hydrogel for multi-modal tumor therapy. Colloids Surf $B$ Biointerfaces. 2021;200:111591. doi:10.1016/j.colsurfb.2021.111591

10. He M, Chen F, Shao D, et al. Photoresponsive metallopolymer nanoparticles for cancer theranostics. Biomaterials. 2021;275:120915. doi:10.1016/j.biomaterials.2021.120915

11. Li J, Zhou C, Zhang J, et al. Photo-induced tumor therapy using $\mathrm{MnO} 2 / \mathrm{IrO} 2-\mathrm{PVP}$ nano-enzyme with TME-responsive behaviors. Colloids Surf B Biointerfaces. 2021;205:111852. doi:10.1016/j. colsurfb.2021.111852

12. Jin F, Liu D, Xu X, et al. Nanomaterials-based photodynamic therapy with combined treatment improves antitumor efficacy through boosting immunogenic cell death. Int $J$ Nanomedicine. 2021;16:46934712. doi:10.2147/IJN.S314506

13. Jiang N, Zhou Z, Xiong W, et al. Tumor microenvironment triggered local oxygen generation and photosensitizer release from manganese dioxide mineralized albumin-ICG nanocomplex to amplify photodynamic immunotherapy efficacy. Chinese Chem Lett. 2021. doi:10. 1016/j.cclet.2021.06.053

14. Kolesnikov IE, Kalinichev AA, Kurochkin MA, et al. Porphyrins as efficient ratiometric and lifetime-based contactless optical thermometers. Mater Design. 2019;184. doi:10.1016/j.matdes.2019. 108188

15. Dias LD, Mfouo-Tynga IS. Learning from nature: bioinspired chlorin-based photosensitizers immobilized on carbon materials for combined photodynamic and photothermal therapy. Biomimetics. 2020;5:4. doi:10.3390/biomimetics5040053

16. Pucelik B, Arnaut LG, Dabrowski JM. Lipophilicity of bacteriochlorin-based photosensitizers as a determinant for PDT optimization through the modulation of the inflammatory mediators. J Clin Med. 2019;9:1. doi:10.3390/jcm9010008

17. Luo T, Nash GT, Xu Z, et al. Nanoscale metal-organic framework confines zinc-phthalocyanine photosensitizers for enhanced photodynamic therapy. $J$ Am Chem Soc. 2021;143(34):13519-13524. doi:10.1021/jacs.1c07379

18. Zhou Z, Song J, Nie L, et al. Reactive oxygen species generating systems meeting challenges of photodynamic cancer therapy. Chem Soc Rev. 2016;45(23):6597-6626. doi:10.1039/c6cs00271d

19. Revuelta-Maza MA, Nonell S, de la Torre G, Torres T. Boosting the singlet oxygen photosensitization abilities of $\mathrm{Zn}$ (ii) phthalocyanines through functionalization with bulky fluorinated substituents. Org Biomol Chem. 2019;17(32):7448-7454. doi:10.1039/ c9ob00872a
20. de Souza JM, de Assis FF, Carvalho CMB, et al. Synthesis of nonaggregating chlorins and isobacteriochlorins from meso-tetrakis(pentafluorophenyl)porphyrin: a study using 1,3-dipolar cycloadditions under mild conditions. Tetrahedron Lett. 2014;55(8):1491-1495. doi:10.1016/j.tetlet.2014.01.049

21. Zhen Z, Tang W, Guo C, et al. Ferritin nanocages to encapsulate and deliver photosensitizers for efficient photodynamic therapy against cancer. ACS Nano. 2013;7(8):6988-6996. doi:10.1021/nn402199g

22. Zhang L, Cui P, Yang H, et al. Metal-organic frameworks as promising photosensitizers for photoelectrochemical water splitting. Adv Sci. 2016;3(1):1500243. doi:10.1002/advs.201500243

23. Stanley PM, Thomas C, Thyrhaug E, et al. entrapped molecular photocatalyst and photosensitizer in metal-organic framework nanoreactors for enhanced solar $\mathrm{CO}_{2}$ reduction. ACS Catal. 2021;11 (2):871-882. doi:10.1021/acscatal.0c04673

24. Hou W, Xia F, Alves CS, Qian X, Yang Y, Cui D. MMP2-targeting and redox-responsive PEGylated chlorin e6 nanoparticles for cancer near-infrared imaging and photodynamic therapy. ACS Appl Mater Inter. 2016;8(2):1447-1457. doi:10.1021/acsami.5b10772

25. Giustarini D, Galvagni F, Tesei A, et al. Glutathione, glutathione disulfide, and S-glutathionylated proteins in cell cultures. Free Radic Biol Med. 2015;89:972-981. doi:10.1016/j.freeradbiomed.2015.10.410

26. Fan H, Yan G, Zhao Z, et al. A smart photosensitizer-manganese dioxide nanosystem for enhanced photodynamic therapy by reducing glutathione levels in cancer cells. Angew Chem Int Ed Engl. 2016;55 (18):5477-5482. doi:10.1002/anie.201510748

27. Shao D, Zhang F, Chen F, et al. Biomimetic diselenide-bridged mesoporous organosilica nanoparticles as an X-ray-responsive biodegradable carrier for chemo-immunotherapy. Adv Mater. 2020;32(50): e2004385. doi:10.1002/adma.202004385

28. Wu M, Meng Q, Chen Y, et al. Large-pore ultrasmall mesoporous organosilica nanoparticles: micelle/precursor co-templating assembly and nuclear-targeted gene delivery. Adv Mater. 2015;27(2):215-222. doi:10.1002/adma.201404256

29. Chen F, Zhang F, Shao D, et al. Bioreducible and traceable Ru(III) prodrug-loaded mesoporous silica nanoparticles for sequentially targeted nonsmall cell lung cancer chemotherapy. Appl Mater Today. 2020;19. doi:10.1016/j.apmt.2020.100558

30. Stöber W, Fink A, Bohn E. Controlled growth of monodisperse silica spheres in the micron size range. J Colloid Interf Sci. 1968;26(1):6269. doi:10.1016/0021-9797(68)90272-5

31. Wan G, Xia H, Wang J, et al. Synthesis of $\mathrm{SiO}_{2} @ \mathrm{SiO}_{2}$ core-shell microspheres using urea-formaldehyde polymers as the templates for fast separation of small solutes and proteins. Chinese Chem Lett. 2018;29(1):213-216. doi:10.1016/j.cclet.2017.06.004

32. Zhang F, Chen F, Yang C, et al. Coordination and redox dual-responsive mesoporous organosilica nanoparticles amplify immunogenic cell death for cancer chemoimmunotherapy. Small. 2021;17(26): e2100006. doi:10.1002/smll.202100006

33. Yang X, Wang D, Shi Y, et al. Black phosphorus nanosheets immobilizing $\mathrm{Ce} 6$ for imaging-guided photothermal/photodynamic cancer therapy. ACS Appl Mater Inter. 2018;10(15):12431-12440. doi:10.1021/acsami.8b00276

34. Yang L, Gao P, Huang Y, et al. Boosting the photodynamic therapy efficiency with a mitochondria-targeted nanophotosensitizer. Chinese Chem Lett. 2019;30(6):1293-1296. doi:10.1016/j. cclet.2019.03.032

35. Wu R, Wang H, Hai L, et al. A photosensitizer-loaded zinc oxidepolydopamine core-shell nanotherapeutic agent for photodynamic and photothermal synergistic therapy of cancer cells. Chinese Chem Lett. 2020;31(1):189-192. doi:10.1016/j.cclet.2019.05.004 


\section{Publish your work in this journal}

The International Journal of Nanomedicine is an international, peerreviewed journal focusing on the application of nanotechnology in diagnostics, therapeutics, and drug delivery systems throughout the biomedical field. This journal is indexed on PubMed Central, MedLine, CAS, SciSearch ${ }^{\mathbb{R}}$, Current Contents ${ }^{\mathbb{R}} /$ Clinical Medicine, $^{2}$
Journal Citation Reports/Science Edition, EMBase, Scopus and the Elsevier Bibliographic databases. The manuscript management system is completely online and includes a very quick and fair peer-review system, which is all easy to use. Visit http://www.dovepress.com/ testimonials.php to read real quotes from published authors. 